\title{
LA NARRATIVA ESPAÑOLA DECIMONÓNICA EN LAS REVISTAS ESTADOUNIDENSES 1875-1900: ANUNCIOS, PUBLICACIÓN Y CRÍTICA
}

\author{
Mercedes Caballer Dondarza
}

\section{RESUMEN}

El objetivo de este trabajo es el de resolver la falta de atención prestada a la narrativa española en la prensa estadounidense durante el último cuarto del siglo XIX. En particular, en las revistas más importantes publicadas tanto en inglés como en español. En ellas hemos hallado anuncios, obras publicadas y críticas de buena parte de las obras decimonónicas de toda la gama de géneros que se cultivan durante esa época. La crítica corre a cargo de plumas españolas y estadounidenses: Leopoldo Alas «Clarín», Emilia Pardo Bazán y Juan Valera entre las primeras, y William D. Howells entre las segundas. Algunas de estas obras y colaboraciones son ya conocidas, otras muchas son inéditas. Se trata de un trabajo puntero en el tema que aúna narrativa y periodismo, y las relaciones entre los principales autores y movimientos literarios españoles y estadounidenses durante una etapa turbulenta entre ambos países.

El reflejo de la actualidad literaria decimonónica española en la prensa estadounidense es prácticamente desconocido. Mucho de este material aún queda por reunir, disperso en publicaciones periódicas coetáneas no sólo españolas sino también extranjeras. Con este estudio se pretende ${ }^{l}$ resolver esa carencia poniendo de manifiesto la presencia de la literatura española, concretamente la narrativa, en Estados Unidos durante el último cuarto del siglo XIX a partir de la revisión de las revistas estadounidenses más relevantes en español y en inglés (facilitada por nuestra residencia en ese país). Nos hemos ceñido a la narrativa española primordialmente porque es ése el género por antonomasia

' A la investigación de este tema dediqué mi tesis doctoral, la narrativa española en la prensa estadounidense (1875-1900), leída en la UNED en 2003 
del último cuarto del siglo que nos ocupa. Es el que sufre un mayor desarrollo y el que encuentra su vehículo ideal en la prensa decimonónica en todas las facetas de la cadena editorial, desde el anuncio a la crítica.

Al iniciar esta investigación surgen una serie de preguntas. En primer lugar, ¿cómo comienza el interés estadounidense por la literatura española, específicamente durante el siglo XIX y cuál es el desarrollo y repercusión de ese interés a lo largo del siglo? ¿coincide el reconocimiento de los autores españoles en la Península Ibérica con aquél de la prensa estadounidense? ¿con qué criterio cuentan los críticos para examinar las obras españolas? ¿se trata de escritores o de simples folletineros (como los llamaría «Clarín»)? ¿afecta el conflicto de 1898 al interés por la literatura española?; para terminar, ¿sería posible establecer la repercusion inmediata y a largo plazo de los autores representados en la prensa estadounidense y para la narrativa española decimonónica, en general? Para dar respuesta a esas preguntas, hemos realizado un vaciado completo de las publicaciones elegidas tanto en inglés como en español, producto del cual es un importante número de anuncios, reseñas, relatos y críticas que conforman el material recogido.

Existe un número de obras publicadas recientemente, en los últimos cinco años, que estudian esa otra prensa denominada con un adjetivo de moda en Estados Unidos, nos referimos a la prensa étnica; si bien, en estas etnias no se hace referencia a los distintos grupos de la población hoy considerada hispana, cuyos antepasados provienen, en algunos casos, directamente de la Península Ibérica. Se reconoce por etnias a los mejicanos, a los puertorriqueños o a los cubanos, entre los hispanos. Y, sin embargo, la mayor parte de la población de Nuevo Méjico se considera a sí misma descendiente de los españoles, incluso en la actualidad. Por último, el reducido número de obras que sí reconoce la participación española en las distintas publicaciones del siglo XIX no otorga especial atención al tema literario. Así, Nicolás Kanellos ${ }^{2}$ sólo lo menciona como uno más de los temas a tratar por estas publicaciones periodicas.

Por otra parte, la bibliografía española revisada presta atención a la prensa estadounidense únicamente en casos aislados como el de Adolfo Sotelo Vázquez ${ }^{3}$ que tiene en cuenta parcialmente la colaboración de "Clarín» en Las Novedades de Nueva York. No se había hecho hasta el momento, por tanto, una revisión exhaustiva de las revistas periódicas estadounidenses decimonónicas a fin de establecer qué autores españoles se veían allí representados, con la contribución al corpus crítico del último cuarto del siglo XIX que esta información pudiera aportar.

La sociedad hispanohablante que vive en los Estados Unidos de finales del siglo XIX comparte con la europea el desarrollo de la actividad editorial. Aunque sería difícil establecer el número de personas que hablan nuestra lengua, porque habría que tener en cuenta los descendientes y los que participan de la cultura española y su lengua como segundo idioma, se produce un cuantioso número de publicaciones en español, que aparece en los Estados Unidos durante las últimas décadas del siglo XIX. Algunas fuentes hacen referencia a más de doscientas cincuenta publicaciones perídicas ${ }^{4}$, mientras que otras reconocen trescientas

${ }^{2}$ Kanellos, Nicolás y Helvetia Martell, (2000), Hispanic Periodicals in the United States, Origins to 1960, A Brief History and Comprehensive Bibliography, Houston: Ane Público Press, Recovering the U.S. Hispanic Literary Heritage.

${ }^{3}$ SotElo VÁzQUEZ, ADOLFo (ed.). (1994), Los artículos de Leopoldo Alas «Clarín» publicados en «Las Novedades», Nueva York, 1894-1897, Cuadernos Hispanoamericanos, Los Complementarios 13/14, junio 1994.

${ }^{4}$ En la cifra de doscientas cincuenta publicaciones se incluyen publicaciones bilingües y políglotas. Las lenguas más usadas son el inglés y el francés, además del español. JAMES F. SHEARER, (1954), «Periódicos es- 
setenta y dos publicaciones ${ }^{5}$ en español. No obstante, esas cifras no tienen en cuenta las publicaciones que aparecen en Nueva York o en Florida. En consecuencia, sería adecuado establecer en tomo a las doscientas publicaciones en español durante el último cuarto del siglo XIX junto a otras mil que se editan en otros idiomas además del inglés (dirigidas a los indios americanos y a los emigrantes alemanes, chinos, escandinavos e italianos).

La labor de divulgación literaria ejercida por las publicaciones decimonónicas es fundamental para el conocimiento y crítica de la literatura de la época, dado que muchas de nuestras obras aparecen por primera vez en este tipo de revistas. En Estados Unidos se produce el mismo fenómeno; la obra Los europeos de Henry James, por ejemplo, aparece en Atlantic Monthly por primera vez entre julio y octubre de 1878 .

En 1830 comienza un período de auge de la prensa periódica estadounidense, debido a una serie de medidas económicas y postales, entre otras, que favorecen a ese sector. En España, en cambio, ese avance llega más tarde. Solamente en un período de cuatro años se triplica el número de periódicos, entre 1878 y 1882 , pasan de casi cuatrocientos a más de novecientos ${ }^{6}$. La prensa española del siglo XIX es el ámbito en el que se da a conocer tanto la literatura española como la extranjera. El caso de Valera, cuya obra, en su mayor parte, se publica en prensa, no es excepcional. Recordemos la opinión de Emilia Pardo Bazán al respecto expresada en el primer número de su Revista de Galicia correspondiente al cuatro de marzo de 1880 :

Las Revistas vienen a ser hoy transacción estipulada entre dos rivales enemigos: el libro y la prensa diaria. En este agitado siglo nuestro, pocas personas disponen de tiempo y constancia suficientes para leer volúmenes: pero, en cambio muchísimas tienen exigencias intelectuales que las publicaciones diarias, al vuelo pensadas y escritas, súbditas de los fugaces intereses e impresiones del momento, no alcanzan a satisfacer. Buena prueba de que el público va pidiendo lecturas más sustanciales que los artículos de fondo y las gacetillas, es el aditamento de hojas semanales a los diarios que más circulan. Va siendo casi tan necesario y grato para la generación actual conocer el juicio de la crítica acerca de los nuevos libros o dramas, ver reseñados los últimos adelantos científicos, leer algún ensayo selecto, alguna bella poesía, como seguir el flujo y reflujo de la política y de las noticias varias. ¡Plegue al cielo que se acentúe y acreciente esta provechosísima tendencia! ${ }^{7}$

pañoles en los Estados Unidos», Revista Hispánica Moderna, núms. 1-2, Enero-Abril: 45-57. Chamberlin, en cambio, hace referencia a « 250 periodicals» publicados en español en Boston, Nueva York, Washington, Filadelfia, Nueva Orleáns, Cayo Oeste y Tampa, y en otras ciudades. Estas revistas incluyen artículos literarios entre otros y son editadas por cubanos, puertorriqueños y ciudadanos de Centro América. En Chamberlin, V.A. e I.A. Schulman, (1976), La Revista Ilustrada de Nueva York, History, Anthology and Index of Literary Selections, Missouri: University of Missouri Press.

${ }^{5}$ Se recoge información de 372 publicaciones de las cuales sólo 136 aparecen en el siglo XIX: 52 en Nuevo Méjico, 38 en Tejas, 34 en California, 11 en Arizona y una en Colorado. Herminio Ríos y Guadalupe Castillo: "Toward a true chicano bibliography» El Grito, A Journal of Contemporay Mexican-American Thought, 3(verano 1970): 17-24; 5(verano 1972): 40-47. En Hutton, F. y Reed B. Straus (eds.), (1995), Outsiders in 19thcentury press history: multicultural perspectives, Ohio: Bowling Green State University Popular Press.

${ }^{6}$ Jesús TIMOTEO ÁlVAREZ: Restauración y prensa de masas: Los engranajes de un sistema (1875-1883), Pamplona, EUNSA, 1992: p. 20, citado en Freire López, Ana $\mathbf{M}^{\mathbf{a}}$, La Revista de Galicia de Emilia Pardo Bazán, (1880) La Coruña: Fundación Pedro Barrié de la Maza, 1999.

${ }^{7}$ Freire López, ANA Mª, (1999), La Revista de Galicia de Emilia Pardo Bazán (1880), La Coruña: Fundación Pedro Barrié de la Maza, p.1. 
A pesar de una extensa y continuada aparición de nuestra literatura en la prensa estadounidense, una de las obras más completas de la historia del periodismo, $A$ history of american magazines $1885-1905^{8}$, tan sólo menciona los nombres de Armando Palacio Valdés en relación con la crítica de William D. Howells, y de Vicente Blasco Ibáñez, que aparece ya en el siglo XX. Más extenso es, en cambio, el espacio dedicado al conflicto cubano y al sensacionalismo que éste despierta ${ }^{9}$.

Aunque el final del siglo XIX representa una etapa turbulenta en la historia entre ambos países, no obstante produce unos resultados satisfactorios en lo que al fomento de nuestra literatura se refiere. A pesar de los acontecimientos, las publicaciones en inglés continúan promocionando obras españolas y trabajos que contribuyen a la enseñanza del español. Ya sea debido a la publicidad producida por el conflicto bélico, o a pesar de ella, lo cierto es que el interés por lo español no decae y las visitas de estadounidenses a nuestro país no dejan de producirse en el último cuarto del siglo XIX, a juzgar por los relatos de viaje realizados.

El interés del estadounidense por la literatura española se ha ido incrementando desde el primer conocimiento de la obra cervantina hasta la literatura contemporánea española. Durante la primera mitad del siglo XIX, Washington Irving escribe obras relacionadas con España ${ }^{10}$. Algunos otros estadounidenses como él viven también en nuestro país por razones profesionales y establecen un vínculo con la cultura de la Península Ibérica que se va desarrollando a medida que ese conocimiento produce curiosidad en otros hispanistas y en el público lector, en general. En la profesión diplómatica, a la que la mayor parte de ese grupo de estadounidenses pertenece, se prefiere el traslado a España antes que a otros países de Europa. Como en el caso de Juan Valera, estos escritores recurren a puestos diplomáticos para mejorar su situación financiera. Se crea así un conocimiento de la cultura y la literatura españolas que crece conforme transcurre el siglo XIX, y del que son herederas las generaciones siguientes de hispanistas.

Teniendo en cuenta que Europa en ese mismo período es testigo del auge de la narrativa y que en España, en particular, la novela realista está en pleno apogeo, compartiendo la última decena del siglo con la debatida novela naturalista española, resulta interesante descubrir hacia dónde se dirige la atención por la literatura española en los círculos literarios estadounidenses que refleja la prensa. Por esa razón hemos distribuido el material recabado siguiendo un orden similar al que tiene lugar tras la producción de una obra literaria, es decir, la promoción de la obra, su divulgación, por entregas en algunos casos, y su correspondiente crítica.

Para lograr nuestro objetivo y tras una exhaustiva revisión de la bibliografía pertinente hallamos siete revistas en inglés y otras tantas en español que representan al panorama periódico en Estados Unidos durante el último cuarto del siglo que nos ocupa. De entre las consideradas para este trabajo, Littell's Living Age es la más antigua. Comienza su edición en 1844 en Boston y tras muchos cambios continúa hasta mediados del siglo XX desde Nueva York. Entre las trimestrales se encuentra North American Review, una de las

${ }^{8}$ Mont, Frank L., (1957), A history of american magazines 1885-1905, Cambridge: Harvard University Press

${ }^{9}$ La historia del periodismo estadounidense más completa tan sólo menciona los artículos en inglés dedicados al conflicto cubano Mott, Frank L., (1962), American journalism, a history of newspapers in the United States through 250 years 1690 to 1940, 2 vols. Nueva York: The Macmillan Company, 3a.ed.

${ }^{10}$ The conquest of Granada (1829), y la colección de cuentos titulada The Alhambra (1832) son algunas de ellas. 
más importantes, que sale de las imprentas bostonianas hasta 1877 , fecha en que se traslada a Nueva York. El escritor e hispanista James R. Lowell es su editor desde 1864, quien ya en ese momento percibe el nuevo ritmo de vida vertiginoso que acusa la sociedad y el papel desfasado que cumple una revista trimestral, por lo que la convierte en mensual.

En 1850 comienza a publicarse una de las más importantes de la época Harper's New Monthly Magazine, que cambiará el futuro de las revistas de ese país. Siete años más tarde se crea Atlantic Monthly, ambas serán las principales representantes de la categoría mensual durante dos décadas, donde la labor editorial corre a cargo de W.D. Howells, entre otros. En 1870 surge su más fiel competidora, la también mensual Scribner's Monthly, antes The Century, que entra en competencia directa con Harper's y Atlantic.

Catholic World y The International Review son publicaciones que llegan a una audiencia menor en Nueva York, la primera mensualmente desde 1865 a 1906 y la segunda bimensualmente entre 1874 y 1878 y mensualmente desde entonces hasta 1883, año final de su existencia. El contrapunto que ofrece una revista católica publicada en inglés con la Revista Católica de Nuevo Méjico resulta de interés para nuestro trabajo. Por su parte, The International Review es una de las publicaciones mencionadas por Mott que publican a autores europeos.

El contenido literario que muestran esas revistas está acorde con la tendencia del momento, es decir, con la narrativa; en particular con la narrativa breve, que resulta perfecta para el espacio que brinda una publicación periódica. Aunque lo que más se vende son las obras seriadas o por entregas y las llamadas «dime novels» (reciben ese nombre por su precio de un «dime» o diez centavos de dólar) que ofrecen un contenido romántico o folletinesco, no obstante, las revistas de reconocida fama como Atlantic, Harper's o Scribner's se reservan el derecho de publicar tales novelas y sólo ofrecen su espacio a una obra de ficción, a veces dos, de autores reconocidos. Son comunes las historias en dos o tres entregas. Por lo general, el contenido literario ocupa un tercio de la revista en aquéllas de importante tirada como las tres anteriores.

Además de la novela, las revistas estadounidenses admiten igualmente otro tipo de trabajos propios de ese tipo de publicaciones, nos referimos a los artículos y editoriales que cubren temas de sociedad, actualidad o crítica literaria, los apartados de promoción y divulgación de obras nuevas con espacios dedicados a las reseñas y anuncios. Los críticos del momento son los mismos escritores ya conocidos por el público que disponen de una buena reputación tales como James Rusell Lowell, Thomas Norton, Henry James, o William D. Howells.

La misma prensa en inglés, recoge algunas quejas por la falta de calidad de las críticas:

We are not well out of the childish age of promiscuous and often silly admiration... the great mischief has always been that whenever our reviewers deviate from the usual and popular course of panegyric, they start from and end in personality, so that the public mind is almost sure to connect unfavorable criticism with personal animosity. Any review thus inspired is worth exactly its weight in Confederate paper. (The Nation julio 6, 1865)."

" «Aún no hemos salido de la edad infantil de la admiración promiscua y, con frecuencia, tonta ... el gran daño ha consistido siempre en que cuando nuestros revisores se desvían del curso de la alabanza de ordinario y popular, ellos empiezan desde y terminan en la personalidad, para estar seguros de que la mente del público establece una conexión entre crítica desfavorable y rencor personal. En consecuencia, cualquier revisión que inspire vale exactamente su peso en papel confederado.». 
Gracias a la información recabada, se pueden establecer varios centros editoriales de publicaciones en español en Estados Unidos. Aquéllas dirigidas a una audiencia hispana (española, mejicana, «nuevomejicana» y sudamericana) surgen en el sur de Estados Unidos en las zonas de una mayor herencia y afluencia de este tipo de población: Califonia (San Francisco y Los Angeles), Florida, Nuevo Méjico y Tejas. En menor medida, también surgen en la Luisiana, donde aparece en 1808 el primer periódico en español: El Misisipi. Como es de esperar, en Nueva York se producen varias publicaciones en nuestra lengua. Recordemos que es el puerto de entrada para refugiados políticos como José Martí, y lugar de reunión para los empresarios hispanos de la época.

Hay una diferencia clara entre la población de habla hispana que llega a refugiarse a Nueva York, o reside ya en ella con anterioridad, y la que se encuentra en Nuevo Méjico. Dado el carácter de puerto de entrada de personas y mercancías, en ella se reúne un buen grupo internacional de intelectuales y comerciantes, que sustentan la labor de aquéllos. Allí, la población, en general, está menos preocupada por la comunidad como espacio físico que, en cambio, es un factor importante en Nuevo Méjico. Por su parte, la población neoyorquina presta más atención al contingente de personas que la componen. De la misma forma, las preocupaciones políticas sobrepasan las fronteras. Este grupo de hispanos posee un sentido más efímero de su paso por Estados Unidos y, por tanto, con mayor apego al país de origen, ya sea Cuba, España o Puerto Rico, principales puntos de procedencia. En consecuencia, las publicaciones que se producen en Nueva York mantienen un carácter más internacional y en ellas encuentran cabida no sólo la literatura española o latinoamericana, sino también la francesa y alemana. Los sucesos más relevantes de cada país encuentran hueco entre sus páginas e incluso, en varias ocasiones, se recolectan fondos para labores intelectuales o humanitarias. Se trata de publicaciones cuyas suscripciones llegan a casi todos los países de Latinoamérica y, desde luego, a España, donde se leen revistas como Las Novedades o La Revista llustrada mencionadas y conocidas por «Clarín», Pardo Bazán y Valera.

La prensa de la zona suroeste de Estados Unidos surge a manos de los mejicanos en tierra aún española, mientras se libra la independencia de la misma entre 1810 y 1821 . Ya en 1810, se editan periódicos en español como La Gaceta y El Mejicano en el virreinato de la Nueva España. Entre las revistas que incluyen información literaria española e hispanoamericana se encuentra el Boletín Popular de Santa Fe, que ve la luz en 1885 y continúa apareciendo hasta 1910. Esta publicación provee de información local, nacional e internacional a la población de Nuevo Méjico. Entre las obras literarias se incluyen tanto las de escritores locales, como las de autores españoles e hispanoamericanos de renombre. Prueba de que la prensa española llega a este lado del océano es un periódico que toma el nombre de otro en Barcelona La Hormiga de Oro (Albuquerque). Junto a los artículos sobre literatura y noticias locales aparecen los correspondientes a historia. Entre los temas tratados se encuentran los de la conquista española y el descubrimiento de América, temas que también se incluyen en la práctica totalidad de las publicaciones en inglés.

En los estudios de la prensa en español, ya señalados, se cree que las voces de las mujeres son minoritarias en las publicaciones del suroeste estadounidense. Sin embargo, como tendremos ocasión de señalar, hay un número cuantioso de obras de escritoras reconocidas en la segunda publicación nuevomejicana elegida. Se trata de la Revista Católica, 
que se publica en Las Vegas, Nuevo Méjico, desde enero de 1875 hasta 1917, a cargo de padres jesuitas. La Revista Católica de Nuevo Méjico es una de las publicaciones que mayor contenido literario aporta a nuestro estudio. Entre las obras allí halladas se encuentran las de autoras como Emilia Pardo Bazán y «Fernán Caballero» y de otras menos reconocidas, como Carmen de Burgos, Eva Canel, Josefa Pujol de Collado, y Luisa Torralba de Martí. Las obras halladas de Carmen de Burgos son inéditas, como también lo son, con toda probabilidad las de aquéllas otras autoras menos reconocidas que aparecen en la Revista Católica. Entre los autores están las obras de algunos menos conocidos por el lector estadounidense dedicados a la novela por entregas como el padre Coloma, Manuel Polo y Peyrolón y Luis Taboada.

Cinco son las revistas procedentes de Nueva York elegidas para nuestro estudio: $\mathrm{La}$ Llumanera de Nova York, Las Novedades, España y los Pueblos Hispano-Americanos, La Ofrenda de Oro, El Progreso, y La Revista Ilustrada de Nueva York. La primera comparte el catalán y el castellano, pero principalmente aquél. El resto se publican únicamente en español aunque al final del siglo se incorporan algunos anuncios también en inglés. Se trata de publicaciones de diferente importancia, algunas como Las Novedades y La Revista Ilustrada mantienen un número de suscriptores considerable en España y en la mayor parte de los países hispanoamericanos. En ambas se mantienen los apartados literarios periódicamente junto a otros de actualidad, culturales e históricos. Entre los colaboradores de Las Novedades y La Revista Ilustrada encontramos a personajes del ámbito político como Emilio Castelar y escritores reconocidos como «Clarín», Emilia Pardo Bazán, Juan Valera y, más tarde, Vicente Blasco Ibáñez. En ese sentido, siguen la línea del periodismo ilustrado español tanto en formato como en contenido. Las otras tres, en cambio, La Llumanera, La Ofrenda de Oro y El Progreso, resultan de menor categoría, aunque no de menor interés, en tanto en cuanto nos permiten establecer cierto equilibrio entre unas y otras. No por ello dejan de ser ilustradas pues también ofrecen a sus lectores algunos grabados.

En la revisión de las revistas ya mencionadas hemos tenido siempre presente que se trata de una etapa difícil debido a la política de independencia de las colonias españolas y el conflicto en el que entran Estados Unidos y España. Con motivo del pasado centenario noventayochista de la pérdida de las últimas colonias españolas, se volvió a plantear la cuestión política del tema. Como se ha venido haciendo en los últimos años, tanto de un lado como del otro del océano Atlántico, la prensa se ha considerado tan culpable del desencadenamiento bélico como algunos de los responsables políticos y militares del momento. Y no solamente se señala a la prensa estadounidense, en especial, al propietario del Journal de Nueva York, el famoso señor William Randolph Hearst ${ }^{12}$, sino también a la prensa española que idealiza la capacidad militar de la Península hasta el final del conflicto.

Un momento delicado en la historia de ambos países que podría haber representado el final de la cultura española en los Estados Unidos y que, sin embargo, la promocionó aún más si cabe. No sólo fueron los artículos, ensayos y reportajes sobre la guerra

${ }^{12}$ Hearst es un periodista ambicioso que inicia su carrera en el Boston Globe como redactor y persigue alcanzar la fama de que disfruta el New York World cuyo propietario es Joseph Pulitzer. Hearst consigue su propósito gracias a un periodismo sensacionalista denominado «amarillo» tras el conflicto cubano, durante el cual obtiene gran éxito. 
de Cuba los protagonistas de los periódicos más sensacionalistas del momento, sino que los anuncios de libros relacionados con Cuba o con España se editan profusamente en los últimos años del siglo XIX ${ }^{13}$. Bien es cierto que el sector liberal y culto estadounidense está en contra de la guerra. Forma parte del mismo una minoría compuesta por personajes conocidos como Mark Twain, el multimillonario Carnegie o el rector de la Universidad de Harvard. Junto a ellos también se oponen a la declaración de guerra, en un principio, algunos empresarios de la costa este que ven sus intereses en la isla y la estabilidad económica del país amenazados por el conflicto. Al sector en contra de la anexión se une el grupo de hispanistas que cultiva el estudio y la enseñanza de la lengua y literatura españolas en la Nueva Inglaterra (constituida por los estados al norte de Nueva York: Connecticut, Maine, Masachusets, Nueva Hampshire, Rhode Island y Vermont) en la que se encuentra uno de los dos focos editoriales más importantes del país. Boston, que aún en la actualidad presume de cuna intelectual, gracias a la universidad de Harvard que alberga, se reparte junto a la ciudad de Nueva York el negocio editorial más importante del momento. Pero la prensa «amarilla» o sensacionalista va dirigida a ese otro sector mayoritario, menos culto, con ánimos imperialistas.

Veamos la explicación que apunta Emilia Pardo Bazán a propósito de dicho adjetivo:

Ocurriósele a un caricaturista yanqui, en momentos de inspiración, representar por medio de un chicuelo antipático y canijo, vestido de amarillo rabioso, al duendecillo de la indiscreción y de la maledicencia, revelador de intimidades de la vida privada; personaje entre imbécil y maligno que pronto se popularizó bajo el nombre de yellow kid. El amarillito hizo escuela y, desde entonces, con el color amarillo se expresó la idea de la prensa chismográfica, pornográfica y puffista o trapalona. A dos diarios de gran circulación que amarillearon, siguieron infinitos, que pulularon y se extendieron por todos los Estados de la Unión americana. (El Liberal, 11 de junio de 1898).

Esos dos diarios a los que hace referencia la autora son, en efecto, los ya mencionados Boston Globe y el New York World, cuyos propietarios son Hearst y Pulitzer, respectivamente. Ese tipo de prensa critica duramente la posición moderada del presidente McKinley al comienzo del conflicto. No obstante, resulta exagerada la afirmación de Raymond Carr ${ }^{14}$ de que el presidente no tuvo otra alternativa que la declaración de guerra, especialmente cuando Estados Unidos aprovechó la ocasión para invadir al mismo tiempo las Filipinas y anexionarse Hawai y la isla de Guam.

Estados Unidos cumple así un objetivo perseguido a lo largo del siglo XIX. Ya en 1823, nos recuerda Félix Santos, escribe John Quincy Adams, entonces secretario de estado, a Hugo Nelson, representante estadounidense en Madrid, que Cuba y Puerto Rico son «apéndices naturales del continente norteamericano» y su anexión «será indispensable para

${ }^{13}$ BaLlow, M.H., (1898) Due south, or Cuba past and present, Boston: Houghton, Mifflin \& Co., en Atlantic Monthly v. LXXXII 1898; Richard Davis, (1898) Cuba in war time. Nueva York: Appleton \& Company en Atlantic Monthly v. LXXXII 1898; Henry Dane, R. Jr., (1898) To Cuba and back, a vacation voyage Boston: Houghton, Mifflin \& Co. en Atlantic Monthly v. LXXXII 1898; Nelan, Ch., (1898), Cartoons of our war with Spain, Nueva York: Frederick A. Stokes Company, en Scribner's Monthly v. XXIV 1898; Ross, C. (1898), Heroes of our war with Spain Nueva York: Frederick A. Stokes Company, en Scribner's Monthly v. XXIV 1898.

${ }^{14}$ Carr, Raymond, (1982), Spain 1808-1975, Oxford: Clarendon Press, $2^{\mathrm{a}}$ ed. 
la existencia e integridad de la Unión». Asimismo, los intentos de compra de la isla se suceden durante los mandatos de los presidentes subsiguientes, Polk, Pierce, Buchanan y Grant, hasta llegar al presidente Cleveland en 1896, quien en su discurso de fin de año expresa una vez más como solución al problema la compra de la isla. Hay que señalar que ese mismo año el partido republicano, con McKinley como representante, organiza una plataforma en favor de la independencia de Cuba. Y al año siguiente, en 1898, ya como presidente, el mismo Mckinley intenta secretamente comprarla en el mes de febrero, algo que España rechaza. Tengamos en cuenta, además, que hacia la mitad del siglo XIX, las relaciones de los exiliados cubanos en Nueva York con sectores comerciales estadounidenses favorecen la labor de los independentistas, entre los que se encuentra José Martí, quien funda junto a Máximo Gómez y Antonio Maceo el Partido Revolucionario Cubano desde esa ciudad en 1892. Esas relaciones, que se estrechan durante los años anteriores a 1898, favorecen la intervención de Estados Unidos en forma de ayuda al sector independentista cubano. De cualquier forma, ese conflicto bélico no interrumpió las relaciones ya establecidas entre intelectuales de ambos países, sino que, por el contrario, éstas se vieron fortalecidas.

Comenzábamos este trabajo preguntándonos por el interés que había despertado la literatura española en Estados Unidos. Protagonistas de dicha atracción y curiosidad por España y lo español son intelectuales, políticos y viajeros, mucho antes de llegar el siglo XIX. Es durante ese siglo, no obstante, y gracias al desarrollo de la prensa periódica, cuando sus colaboradores expresan esa atracción por lo exótico y lo bárbaro, si se nos permite utilizar dicho término romántico, que representa lo español para algunos de ellos. Gracias al conocimiento de diversos intelectuales estadounidenses que cruzan el océano por otras razones que las propiamente literarias, se consigue transformar esa imagen de lo español, al menos entre una minoría. El legado de esos individuos entre los que se encuentran Washington Irving, George Ticknor, Henry W. Longfellow, James Russell Lowell y William Dean Howells es de notable importancia para cualquier investigador del tema. Debemos incidir, concretamente, en el legado de dos de ellos: George Ticknor y William D. Howells. Recordemos que Ticknor es el primer estadounidense que realiza un viaje a España con la intención de conocer y estudiar la literatura de nuestro país, después de haber sido elegido profesor de lengua y literatura españolas en la Universidad de Harvard en 1819 , posición que ocupa hasta 1836 cuando le cede el puesto a Longfellow.

Ya durante el siglo XIX se reconoce la atracción por lo español desde un comentario en la prensa que menciona a un número mayor de intelectuales del que hayamos podido considerar en este trabajo, donde hemos tenido en cuenta primordialmente aquéllos cuya influencia para la literatura española en Estados Unidos resulta digna de especial mención y detenimiento:

It is curious what an attraction Spain and Spanish history have always had for the best Americans. It is, as Hawthorne once said, as if America wished to pay the debt she owed for her discovery. Prescott and Motley, Washington Irving and Longfellow, have each in turn caught inspiration from the history or legends of Spain..$^{15}$

15 «Es curiosa la atracción que sienten los mejores americanos por España y la historia española. Es, como Hawthorne dijo una vez, como si América quisiera pagar la deuda que le debía por su descubrimiento. Prescott y Motley, Washington Irving y Longfellow, cada uno a su vez se ha inspirado en la historia o las leyendas de España.» «Life, letters, and journal», Living Age I: 508. 
Después de considerar varios testimonios, tanto de ciudadanos de a pie como de escritores e intelectuales de finales del siglo XIX, se puede concluir que la inclinación que se muestra por la lengua española en los círculos cultos de la época que la practican continúa en aumento a lo largo del siglo. La opinión ofrecida por Carlos Bransby (traductor de la novela de «Fernán Caballero» Un servilón y un liberalito o tres almas de Dios para la editorial bostoniana D.C. Heath en 1909) a Las Novedades de Nueva York justifica el número creciente de anuncios de la enseñanza del español y la incorporación de nuestra lengua a las universidades estadounidenses. Pero no son sólo las razones culturales las que promueven una mayor demanda de nuestra lengua. La apertura comercial al resto del continente americano, ya durante el siglo XIX, juega lógicamente un importante papel. De igual manera y de forma lamentable en algunos casos, el conflicto cubano contribuirá al interés por lo español de algunas publicaciones.

Colaboraciones como la entablada por Valera con la editorial Appleton de Nueva York, durante su estancia en Washington como diplomático entre 1883 y 1886 , son hechos notorios a tener en cuenta en el avance y desarrollo de ese interés por nuestra cultura. Las aspiraciones valerianas con la casa Appleton, no obstante, quedan, si se nos permite la expresión, en agua de borrajas. El inicio de un negocio con esa editorial neoyorquina que habría aportado muchos y diferentes beneficios a la literatura española del momento no llega a cuajar, al menos no con esa editorial, a excepción de las obras de Valera ${ }^{16}$. Sin embargo, a Palacio Valdés, que goza de gran popularidad entre el lector es. tadounidense, según se puede apreciar por las ediciones de sus obras con otras editoriales neoyorquinas ${ }^{17}$, no parece haberle afectado ese hecho. Como tampoco resulta perjudicada la relación comercial establecida entre la Pardo Bazán y la editorial Cassell, ya que ésta traduce y publica buen número de sus obras ${ }^{18}$. Otros autores como Clarín o Pe-

16 VAlera, JuAn, (1886) Pepita Ximénez the spanish of Juan Valera with and introduction by the author written specially for this edition, Nueva York: Appleton \& Company.; (1891) Don Braulio, traducción de Clara Bell, Nueva York: Appleton.; (1891) Doña Luz, traducción de Mary J. Serrano, Nueva York: Appleton, con introducción recogida en Harper's Magazine: "Valera and Doña Luz" por W.D. Howells, Colección: Appletons' town and country library; (1898) Pepita Ximenez, nueva edición con introducción del autor, Nueva York: D. Appleton; (1908) Pepita Jiménez, Death's Modern Language Series editada con notas y vocabulario por G.L. , Lincoln/Harvard University. Boston: Heath \& Company.

${ }^{17}$ Palacio Valdés, Armando (1886), The Marquis of Peñalta, a realistic social novel, Dole, Nathan Haskell, (trad.); 1852-1935, Nueva York. T.Y. Cromwell \& Co; (1888), Maximina y Don Armando Palacio Valdés, Dole, Nathan Haskell 1852- (trad.) Nueva York: Thomas Y. Cromwell; (1890), Scum, Nueva York: United States Book Co., colección: Lovell's foreign series; (1892), Faith, Hapgood, Isabel Florence, (trad.)18501928, Nueva York: Cassell publishing company; (1894), The origin of thought, Isabel Florence (trad.) 1850-1928, Nueva York: I.F. Hapgood, se incluye un extracto de la crítica de Cosmopolitan Magazine de 1894; (1895), The grandee, Nueva York: Geo. Gottsberger Peck; (1900) The joy of captain Ribot, trad. Minna Caroline Smith, Nueva York; (1900) José, ed. FJ.A. Davidson, Boston: D.C. Heath \& Company, colección: Heath's modern language series, editada con vocabulario de Alice P. Hubbard; (1901) José, trad. Minna Caroline Smith, Nueva York; (1906) La alegría del capitán Ribot ed. Morrison y Churchman, Boston.

${ }^{18}$ Pardo Bazán. Emilia, (1890), Russia, its people and its literature, trad. F.H. Gardiner, Chicago: A.C. McClurg \& Company; (1891), A christian woman, Springer, Mary (trad.), Nueva York: Cassell publishing company; (1891), Morriña (Homesickness), Serrano, Mary Jane Christie (trad.) Nueva York: Cassell Publishing Company; (1891), The swan of Vilamorta, trad. Mary J. Serrano, Nueva York: Cassell Publihing Company; (1891), A wedding trip, Serrano, Mary Jane Christie (trad.), Chicago : E. A. Weeks; (1891), A wedding trip, Serrano, Mary Jane Christie (trad.), Nueva York: Cassell Publishing Company; (1891), The angular stone, Mary J. Serrano (trad.) Nueva York: Cassell publishing company; (1892), The angular stone, Serrano, 
reda resultan, en cambio, menos afortunados al respecto, puesto que son prácticamente ignorados por las casas editoras estadounidenses.

Es destacable en efecto, que se produzca una clara coincidencia temporal entre la aparición de los comentarios y críticas de Howells de 1886 a 1892 en la Harper's New Monthly y la publicación y traducción de las novelas de Emilia Pardo Bazán y de Benito Pérez Galdós. La primera vez que Howells menciona a esa autora en su espacio crítico es en 1890 con motivo de la publicación de Morriña en España el año anterior. En 1891, un año después de esa crítica, se traduce Morriña y la editorial Cassell la publica. Durante ese mismo año de 1891 aparecen las traducciones de El cisne de Vilamorta y de Una cristiana, a las que Howells dedica también su espacio en la Harper's New Monthly. Por tanto, este hispanista examina esas obras antes de su traducción en el caso de Morriña y después de la misma en el de las otras dos. Si la traducción de Morriña está relacionada o no con ese comentario de Howells es difícil de establecer. Desconocemos la fecha exacta en la que doña Emilia entra en contacto con la editorial Cassell. Solamente hemos podido acudir al comentario encontrado al respecto que realiza la autora en «Ángel Guerra» y publicado en Nuevo Teatro Crítico ese mismo año de $1891^{19}$. De dicho comentario podemos deducir que la visita del «extranjero distinguido», enviado por la editorial mencionada, se habría producido en un pasado no muy lejano con respecto al comentario de doña Emilia, con probabilidad durante 1890 . En ese caso, el interés de Cassell por las obras pardobazanianas podría estar relacionado con los comentarios de Howells realizados antes de la traducción de éstas.

En lo que se refiere a Galdós también encontramos las dos situaciones: La familia de León Roch se publica en 1888 y la crítica de Howells aparece a posteriori ese mismo año, mientras que la crítica a la traducción de Doña Perfecta se produce a priori, es decir, antes de salir al mercado su traducción, lo que no resulta sorprendente si se tiene en cuenta que el artículo de Howells titulado «A great novel» aparecido en Harper's Bazar se incorporará a la misma y la editorial que la publica es Harper \& Brothers. En este caso no podríamos establecer relación alguna con la crítica de Howells y la traducción de las obras galdosianas puesto que la primera aparece a posteriori.

Ha sido de gran interés examinar y comparar las distintas formas y expresiones que adopta la narrativa española en las publicaciones periódicas elegidas. El negocio editorial

Mary Jane Christie (trad.), Nueva York: Mershon Company; (1900), Secret of the yew tree or A christian woman, M. Springer (trad.), Nueva York: The Mershon company; (1900), A Galician's girl romance (Morriña) Mary Serrano (trad.), Nueva York: The Mershon Company; (1900), Pascual López, con notas en inglés y vocabulario por el profesor W.I. Knapp, Phd. LL D., Nueva York: Ginn and Company; Plumber, Mary Wright (ed.), (1899, 1900) Contemporary Spain as shown by her novelists 1856-1916, a compilation by Mary Wright Plumber, with aan introduction by Edward E. Hale, comp.; Alarcón. Pedro Antonio de; 1833-1891 . ; Pardo Bazán, Emilia; condesa de,; 1852-1921; Pérez Galdós, Benito; 1843-1920.; Palacio Valdés, Armando; 1853- ; Valera y Alcalá Gallano, Juan; 1824-1905. Nueva York: Truslove; London: Hanson \& Comba.; Warner, Ch. D. (ed.), (1896), Library of the world's best literature: ancient and modern. v.XIX, Nueva York: R.S. Peale.

${ }^{19}$ Un distinguido extranjero que vino a Madrid a cerrar contratos con los novelistas españoles, para que sus obras se publiquen traducidas en los Estados Unidos, me decía que, en opinión de Zola, nuestra novela actual es la tercera en mérito de las que hoy se conocen en Europa: el autor de Germinal reservaba, por supuesto, la primicia a la novela francesa y a la rusa, y no estando resuelto cúal de estas dos triunfa, la nuestra vendría a ser la segunda realmente. Ángel Guerra» (1891) Nuevo Teatro Crítico, agosto, en Sotelo Vázquez, M. (1990), Ángel Guerra de Benito Pérez Galdós y sus críticos (1891), Barcelona: PPV, Literatura y Pensamiento en España (siglos XVIII-XIX-XX). 
que se deriva de la publicación de las revistas en inglés más importantes del momento como la Atlantic Monthly, la Harper's New Monthly Magazine, la North American Review y la Scribner's Monthly permite una nueva valoración del comportamiento de las obras literarias españolas en el mercado estadounidense. Los anuncios y reseñas de esas obras constituyen el primer eslabón de la cadena. La promoción de las obras está estrechamente ligada al negocio de las editoriales, dueñas también de las revistas mencionadas. Aunque no son sólo las obras reconocidas por la prensa estadounidense las que llegan a salir al mercado. Si exceptuamos el caso de Armando Palacio Valdés, cuya amistad con William Dean Howells, el crítico estadounidense por antonomasia, le proporciona un buen escaparate para sus obras, parece ser más importante la relación del autor con la propia editorial, como ocurre con doña Emilia, que la publicidad que reciban sus obras a través de anuncios, reseñas y críticas. Así, las novelas pardobazanianas que comenta Howells son pocas comparadas con las que se traducen y publican en Estados Unidos. No obstante, la crítica emitida por Howells guarda una relación importante con la etapa en la que se publican y traducen esas obras y probablemente tenga que ver con el inicio del proceso.

El sorprendente número de publicaciones periódicas en español que se editan en Estados Unidos durante el último cuarto del siglo XIX, más de doscientas, como hemos mencionado, ejerce un importante papel en el mantenimiento y desarrollo de la atención prestada a la literatura española en ese país. Aunque las publicaciones en español también participan de ese negocio editorial, en su caso representa un recurso inevitable para subsistir. Algunas, no obstante, poseen los medios necesarios para no tener que acudir a los anuncios, concretamente la Revista Católica de Nuevo Méjico. Por su parte, la otra revista nuevomejicana revisada, el Boletín Popular, ofrece anuncios comerciales, pero no literarios. Las Novedades y La Revista llustrada de Nueva York, en cambio, se ven obligadas a ofrecer anuncios y libros a la venta, así como los servicios de imprenta necesarios para el cliente, en el caso de la primera. Además, Las Novedades es la única publicación, de entre las examinadas, que incluye de manera fija una sección dedicada a las «Notas bibliográficas» y a los «Libros Nuevos», donde se comentan las novedades editoriales. Es allí, por tanto, donde se muestran unas cuantas reseñas de obras de Alarcón y Valera y se ponen a la venta la mayor parte de las obras contemporáneas.

Las publicaciones periódicas son plataformas anunciadoras de las nuevas obras que, en muchos casos, vende la propia empresa editora de la revista. Las críticas de esas obras por parte de autores reconocidos favorecen el conocimiento de las mismas y, por supuesto, las ventas. Comienza así una actividad mercantil en torno a la literatura. No obstante, la labor de divulgación que se ejerce a través de la promoción de las obras españolas es fundamental. Las reseñas son meros anuncios descriptivos o resúmenes de carácter comercial y sensacionalista de las obras en cuestión, especialmente en las publicaciones en inglés. No obstante, en las de mayor envergadura como la Atlantic Monthly, la Harper's New Monthly Magazine y la Scribner's Monthly se promocionan las obras de Pedro Antonio de Alarcón, Gustavo Adolfo Bécquer, Benito Pérez Galdós y Armando Palacio Valdés. En cambio, se ignoran las obras de Leopoldo Alas, José $\mathbf{M}^{a}$ de Pereda, Emilia Pardo Bazán y Juan Valera. Por tanto, gracias a la revisión del material hallado hemos podido observar el trato prioritario de algunos escritores frente a otros.

En las revistas examinadas, los anuncios y reseñas no siguen un orden coherente con la aparición de las obras en España. Además, las obras elegidas no son tampoco las 
que más éxito consiguen en nuestro país y llegan a la prensa estadounidense, en los mejores casos, con una década de retraso, en los peores con dos. Hecho que resulta comprensible si consideramos el retraso de, al menos, una década desde la aparición y publicación de las principales obras realistas y naturalistas estadounidenses con respecto a las españolas. Lo que no quiere decir que no hubiese obras realistas con anterioridad en ese país, si bien las más importantes entre las que se encuentran Silas Lapham de Howells, The bostonians de James o The adventures of Huckleberry Finn de Twain aparecen mediada ya la década de 1880 . Si además tenemos en cuenta el ya mencionado papel influyente de la crítica de Howells, cuya labor más importante aparece en la Harper's New Monthly Magazine entre 1886 y 1892, no resulta sorprendente que la mayor parte de la narrativa española decimonónica reciba su crítica entonces.

El segundo eslabón en la cadena editorial está representado por la publicación de la narrativa en las revistas de aquel país. Obras de autores que pertenecen a una generación anterior a la que cultiva el Realismo, como «Fernán Caballero», acaparan muchos números de la Revista Católica de Nuevo Méjico. Autoras menos conocidas, como Carmen de Burgos o «Raquel» y Luisa Torralba de Martí o «Aurora Lista», también ven sus novelas y relatos breves publicados en esa revista, junto a autores ya reconocidos como el padre Luis Coloma, Manuel Polo y Peyrolón y Emilia Pardo Bazán. De esta última también se publica su novela La piedra angular en Las Novedades de Nueva York entre 1892 y 1893 y su cuento «Sédano» en La Revista Ilustrada de Nueva York, ambos en fechas muy cercanas a su aparición en España. En la Revista Católica, en cambio, las novelas y relatos breves aparecen entre 1886 y 1900 , cuando han pasado unas cuantas décadas desde que se publicaran las primeras obras de «Fernán Caballero» en nuestro país. Sin embargo, las obras contemporáneas se incluyen en esa revista con bastante más rapidez, que oscila entre uno y diez años. Existe una pervivencia clara, por tanto, del interés por la literatura romántica y por aquélla de tono religioso en una revista como la mencionada.

También en la prensa en español hallamos colaboraciones de los autores más representativos de la narrativa española decimonónica. Emilio Castelar y Leopoldo Alas «Clarín» participan en Las Novedades de Nueva York de manera asidua. En el caso de Clarín se trata de colaboraciones de carácter crítico porque contienen una serie de juicios acerca de algunas obras y sus correspondientes autores. Ya examinadas por Adolfo Sotelo Vázquez entre los años 1894 y 1897, hemos podido adelantar el período a 1892 con la serie inédita titulada «Como gustéis». Por su parte, Emilia Pardo Bazán y Juan Valera contribuyen con sus escritos a La Revista Ilustrada de Nueva York. Se trata de colaboraciones de carácter cultural en las que se registran los últimos acontecimientos editoriales, literarios y sociales del momento, estableciendo un lazo de unión importante entre España, los países latinoamericanos y Estados Unidos.

En,la prensa en inglés, concretamente en Littell's Living Age localizamos un número abundante de relatos breves de Pedro Antonio de Alarcón traducidos exclusivamente para esa revista entre 1897 y 1899 , también unas cuantas décadas después de su aparición en España. La misma revista publica varios relatos de Emilia Pardo Bazán, algunos dedicados a la mujer como «The women of Spain», que luego verá la luz en nuestro país, concretamente un año después, en La Época. Hecho notorio sin precedente, al menos entre las publicaciones revisadas. Parece que en un intento de evitar los comentarios críticos al respecto, doña Emilia se adelante justificando esa preferencia ofrecida al lector británico y así lo explica en la versión española. 
La narrativa española decimonónica que se publica en la prensa estadounidense es de carácter breve: cuadros de costumbres, novela corta, novelitas, relatos breves, cuentos, artículos, críticas. Todos ellos encuentran cabida en el formato de la revista ya sea en uno solo de sus números o por entregas. La prensa constituye el formato idóneo para este tipo de narrativa que hemos hallado principalmente en las publicaciones estadounidenses en español. Por contra, las publicaciones en inglés prefieren a los autores estadounidenses que cultivan también la narrativa breve en la que aún se incluyen folletines románticos junto a obras de un realismo moderado o de costumbres. Este último es cultivado por las escritoras estadounidenses y se correspondería con el que practica ya Fernán Caballero en torno a la mitad del siglo.

Autores reconocidos del momento, como Leopoldo Alas «Clarín» o José $\mathrm{M}^{\mathrm{a}}$ de Pereda, resultan de segunda fila en los Estados Unidos, a juzgar por el mínimo espacio que se les dedica en la prensa de ese país, a excepción de las colaboraciones clarinianas, si se tiene en cuenta el auge que experimentan otros como Armando Palacio Valdés, cuyas obras obtienen un reconocimiento y promoción mayor del que podría esperarse. No obstante, en el caso de Pereda, este hecho se puede atribuir a su desconocimiento por parte de Howells, principal crítico del momento. Lo propio sucede con «Clarín» cuyas obras no llegan a los Estados Unidos, aunque Howells lo menciona en el capítulo dedicado a Benito Pérez Galdós en su obra Literary friends and acquaintances, en referencia con el origen de la novela realista. Por consiguiente, sabemos que Howells conoce a Clarín y no podemos descartar que hubiera leído sus obras en español.

Las obras narrativas de los autores mencionados que se editan en la prensa estadounidense distan, en algunos casos, de ser las que gozan de mayor reconocimiento en España. Así, se publican y traducen novelas del calibre de Doña Perfecta de Pérez Galdós o Pepita Jiménez de Valera, junto a la mayor parte de las obras de Palacio Valdés. En cambio, se ignoran otras obras de esos mismos autores, especialmente las que contienen ya elementos naturalistas como La desheredada de Pérez Galdós o La Tribuna de Emilia Pardo Bazán y las muchas otras que aparecen con posterioridad a ésas.

Por su parte, la crítica de las publicaciones en inglés se ciñe, casi exclusivamente, a la realizada por William Dean Howells en la Harper's New Monthly Magazine. Su labor es imprescindible para entender el reconocimiento de autores como el ya mencionado Palacio Valdés junto a Pardo Bazán, Pérez Galdós y Valera. Gracias al examen del conjunto de obras publicadas en la prensa estadounidense y de las críticas que éstas reciben se logra responder a las dudas acerca de la elección y reconocimiento, por parte de la prensa estadounidense, de autores y obras españolas determinadas y los criterios seguidos para ello.

La principal diferencia que hemos encontrado entre las narrativas estadounidense y española es el retraso con el que aparecen el Realismo y el Naturalismo en la primera y más aún, la falta relativa de impacto y polémica que el Naturalismo suscita en los Estados Unidos cuando se compara con lo sucedido en España. Directamente relacionada con ese reconocimiento, por parte de Howells, de la narrativa española está la desvinculación de los autores y críticos estadounidenses que colaboran en las publicaciones examinadas con respecto al movimiento naturalista. Ello puede deberse, por una parte, a la influencia puritana aún fuertemente arraigada en los círculos literarios tradicionales en zonas del noreste estadounidense, que lleva a algunos críticos a acusar a las 
obras naturalistas de demasiado «colorismo». No obstante, se aceptan las dudas religiosas que reflejan algunas de las novelas españolas publicadas en la prensa como $\mathrm{Pe}$ pita Jiménez o La hermana San Sulpicio y sus finales. Y por otra, al hecho de que los autores estadounidenses que cultivan el naturalismo, como Stephen Crane, Jack London o Frank Norris, lo hagan con retraso, ya que sus obras salen a la luz durante la década de 1890. Además, las reminiscencias románticas que aparecen en esas novelas españolas y la sensualidad que algunas de las descripciones ofrecen atraen a un público acostumbrado a la novela por entregas.

La revisión de la narrativa decimonónica aparecida en la prensa estadounidense del momento muestra cuáles fueron las preferencias de lectores, editores, y críticos del momento. Información que prueba la importancia de la prensa en general como fuente para la investigación literaria, como muestran otros estudios recientes de las publicaciones nacionales y extranjeras del siglo XIX. Dado el desconocimiento actual de la presencia de la narrativa española en la prensa estadounidense, y en las publicaciones en español, en particular, este trabajo abre nuevos caminos de investigación sobre el tema.

En nuestro estudio hemos sacado a la luz algunas participaciones, hasta el momento desconocidas, de autoras españolas en la prensa estadounidense revisada, como las de Carmen de Burgos, Eva Canel, o Emilia Pardo Bazán. Hemos reconsiderado las fechas de otras participaciones como la de Leopoldo Alas «Clarín» en Las Novedades de Nueva York que comienza en 1892, anterior a la ya determinada de 1894 , que contribuye a completar su ya extensa labor crítica. Por último, hemos reconocido y considerado la importancia de las relaciones personales entre intelectuales de ambos países, como las de Howells y Valera, o Howells y Palacio Valdés, que ya otros habían adelantado, examinando la repercusión de esas amistades a través de la crítica y reconocimiento de Howells, cuya labor es elemental en la promoción de la literatura española en Estados Unidos. En suma, en la prensa estadounidense examinada se han hallado ejemplos de la narrativa española decimonónica adscrita sobre todo al Romanticismo y al Realismo y, en menor medida, al Naturalismo. Comienza así el siglo XX con la continuación de un interés justificado por España y su literatura, a pesar o gracias al conflicto bélico a caballo entre los dos siglos. 